\title{
SPECIAL ARTICLE Pre-Vent: the prematurity-related ventilatory control study
}

Phyllis A. Dennery ${ }^{1}$, Juliann M. Di Fiore ${ }^{2}$, Namasivayam Ambalavanan ${ }^{3}$, Eduardo Bancalari ${ }^{4}$, John L. Carroll ${ }^{5}$, Nelson Claure ${ }^{4}$, Aaron Hamvas ${ }^{6}$, Anna Maria Hibbs ${ }^{2}$, Premananda Indic ${ }^{7,8}$, James Kemp ${ }^{9}$, Katy N. Krahn ${ }^{10}$, Douglas Lake ${ }^{10}$, Aaron Laposky ${ }^{11}$, Richard J. Martin ${ }^{2}$, Aruna Natarajan ${ }^{12}$, Casey Rand ${ }^{6}$, Molly Schau ${ }^{6}$, Debra E. Weese-Mayer ${ }^{6}$, Amanda M. Zimmet ${ }^{10}$ and J. Randall Moorman ${ }^{10}$

BACKGROUND: The increasing incidence of bronchopulmonary dysplasia in premature babies may be due in part to immature ventilatory control, contributing to hypoxemia. The latter responds to ventilation and/or oxygen therapy, treatments associated with adverse sequelae. This is an overview of the Prematurity-Related Ventilatory Control Study which aims to analyze the underutilized cardiorespiratory continuous waveform monitoring data to delineate mechanisms of immature ventilatory control in preterm infants and identify predictive markers.

METHODS: Continuous ECG, heart rate, respiratory, and oxygen saturation data will be collected throughout the NICU stay in 500 infants $<29$ wks gestation across 5 centers. Mild permissive hypercapnia, and hyperoxia and/or hypoxia assessments will be conducted in a subcohort of infants along with inpatient questionnaires, urine, serum, and DNA samples.

RESULTS: Primary outcomes will be respiratory status at $40 \mathrm{wks}$ and quantitative measures of immature breathing plotted on a standard curve for infants matched at 36-37 wks. Physiologic and/or biologic determinants will be collected to enhance the predictive model linking ventilatory control to outcomes.

CONCLUSIONS: By incorporating bedside monitoring variables along with biomarkers that predict respiratory outcomes we aim to elucidate individualized cardiopulmonary phenotypes and mechanisms of ventilatory control contributing to adverse respiratory outcomes in premature infants.

Pediatric Research (2019) 85:769-776; https://doi.org/10.1038/s41390-019-0317-8

\section{INTRODUCTION}

Pulmonary complications of prematurity represent leading causes of infant mortality, morbidity, and lasting disability. Though prematurity-related neonatal mortality and other morbidities have declined in recent decades ${ }^{1}$ the incidence of bronchopulmonary dysplasia (BPD), defined as oxygen requirement at or beyond 36 weeks corrected age $^{2}$ has not, leading to significant morbidity and mortality. Despite the introduction of exogenous surfactant therapy to address respiratory distress syndrome of prematurity and the widespread availability of non-invasive respiratory support methods in neonatal intensive care units worldwide, chronic respiratory illness persists until hospital discharge and beyond in a substantial percentage $(66 \%)^{3}$ of very premature infants. The use of supplemental oxygen to treat frequent episodes of intermittent hypoxemia $(\mathrm{IH})$ during the first few weeks after birth could potentially contribute to hyperoxemiainduced lung disease. ${ }^{4}$ Hypoxemia could result from disordered ventilatory control secondary to attenuated or aberrant peripheral and/or central chemoresponsiveness to perturbation, volutrauma due to mechanical ventilation or both.

Respiratory morbidity in preterm infants stems from pathologies of airway, parenchyma, vasculature, and innervation. This already complex picture is rendered more so by the fact that control of breathing by the central and autonomic nervous systems are not fully developed in the premature infant. ${ }^{5}$ Central apnea may also drive the requirement for oxygen therapy. Respiratory insufficiency in a premature neonate thus presents a multi-dimensional phenotype that is difficult to deconstruct and cohesively describe for bedside clinicians and scientists alike.

Extreme prematurity predisposes to both apnea of prematurity (AOP), defined as a cessation of breathing for $\geq 20 \mathrm{sec}$ or a shorter pause accompanied by bradycardia, cyanosis, or pallor, ${ }^{6}$ as well as pulmonary morbidity and neurodevelopmental impairments. Though specific causality among these three conditions is not fully elucidated, a control of breathing deficit may predispose

\footnotetext{
${ }^{1}$ Hasbro Children's Hospital, Warren Alpert School of Medicine, Brown University, Providence, RI, USA; ${ }^{2}$ Department of Pediatrics, Division of Neonatology, University Hospitals: Rainbow Babies \& Children's Hospital, School of Medicine, Case Western Reserve University, Cleveland, OH, USA; ${ }^{3}$ Department of Pediatrics Molecular and Cellular Pathology, and Cell Developmental and Integrative Biology, Division of Neonatology, University of Alabama at Birmingham School of Medicine, Birmingham, AL, USA; ${ }^{4}$ Department of Pediatrics, Division of Neonatology, Holtz Children's Hospital_Jackson Memorial Medical Center, University of Miami Miller School of Medicine, Miami, FL, USA; ${ }^{5}$ University of Arkansas for Medical Science, Department of Pediatrics, Little Rock, AR, USA; ${ }^{6}$ Northwestern University Feinberg School of Medicine, Ann \& Robert H. Lurie Children's Hospital of Chicago, Stanley Manne Children's Research Institute, Chicago, IL, USA; ${ }^{7}$ Department of Electrical Engineering, University of Texas at Tyler, Tyler, TX, USA; ${ }^{8}$ Department of Pediatrics, Division of Neonatology, University of Alabama at Birmingham School of Medicine, Birmingham, AL, USA; ${ }^{9}$ Department of Pediatrics, Washington University School of Medicine in St. Louis, St. Louis, MO, USA; ${ }^{10}$ Division of Cardiovascular Medicine, University of Virginia School of Medicine, Charlottesville, VA, USA; ${ }^{11}$ National Institute of Health, National Center of Sleep Disorders Research, Bethesda, MD, USA and ${ }^{12}$ Division of Lung Diseases, National Heart Lung and Blood Institute, National Institute of Health, Bethesda, MD, USA Correspondence: Phyllis A. Dennery (phyllis_dennery@brown.edu)
}

Received: 25 October 2018 Revised: 10 January 2019 Accepted: 16 January 2019

Published online: 1 February 2019 
to AOP and pulmonary disease. The most widely used methylxanthine, caffeine, effectively reduces apnea incidence ${ }^{7}$ but does not always reduce the frequency or severity of hypoxemic episodes from apnea or periodic breathing. ${ }^{8}$

Taken together, immature respiratory control superimposed on underdeveloped lungs can result in sufficient apnea or periodic breathing to necessitate oxygen therapy at 36 weeks, meeting the criteria for diagnosis of BPD. In the Prematurity and Respiratory Outcomes Program (PROP) trial, $40 \%$ of BPD infants earned their oxygen requirement from unstable breathing patterns that were unmasked when oxygen was removed. ${ }^{9}$ Such abnormal control of breathing requiring oxygen therapy can have long-term impact on lung and neurological function ${ }^{10}$ and maturation, with resultant prolonged hospitalization and economic burden.

These observations point to a need for quantifying control of breathing in premature infants as both a clinical tool for their care as well as a research metric for studying the relationship between abnormal control of breathing, its therapy, developmental trajectory, and clinical outcome. While NICU bedside cardiorespiratory monitoring is effective in highlighting immediate and short-term physiologic perturbations, the ability to quantify trajectories and patterns of events over weeks or months, and understand the long-term impact on outcomes remains a challenge. Because the development of the autonomic nervous system accelerates during late pregnancy, infants delivered before the third trimester lack the physiologic maturity required for uninterrupted breathing often resulting in central apnea and periodic breathing with recurrent hypoxemia and bradycardia.

The Prematurity-Related Ventilatory Control (Pre-Vent) study is an $\mathrm{NIH}$-funded cooperative agreement among 6 academic centers with the primary goal of elucidating the role of ventilatory control as a determinant of respiratory dysfunction in preterm infants. PreVent includes a single multicenter protocol to develop a predictive model of respiratory outcomes, and 5 center-specific protocols testing hypotheses regarding control of breathing mechanisms, in premature infants. Additionally, a DNA biorepository will be created. In all, more than 500 premature infants will be studied.
This paper describes the study, its anticipated data sets, and expected contributions to predicting respiratory outcomes.

\section{METHODS/STUDY DESIGN}

Organizational structure

The Pre-Vent organizational structure is shown in Fig. 1. The Steering Committee comprises an external Chair appointed by the National Heart, Lung, and Blood Institute (NHLBI), co-PIs from each Center and the Leadership and Data Coordinating Center (LDCC), and the NHLBI Program Scientist and Program Officer.

The role of the Steering Committee is to coordinate and oversee the Multicenter Study and harmonize and synergize the Clinical Research Center (CRC) Studies. The NIH-appointed Observational Safety Monitoring Board includes experts in neonatology, autonomic medicine, ventilatory control, intensive care, bioethics, and biostatistics and provides independent ethical and safety oversight at regular intervals, presided over by the Executive Secretary: a member of NHLBI Program Staff.

Leadership Data Coordinating Center (LDCC). The roles of the LDCC at the University of Virginia (UVA) are to organize and oversee the regulatory and logistical aspects of the multicenter protocol, to assist centers in collecting and storing cardiorespiratory signals from bedside monitors and to propose and implement the multicenter protocol to quantify disordered breathing. The LDCC Clinical Trials Office manages the clinical database including designing the electronic case report forms, providing support for standardization of definitions, training Center Research Coordinators in clinical data collection, and performing regular data quality monitoring and analysis. The LDCC Biorepository and Tissue Research Facility receives and stores buccal swab specimens and extracts, quantifies, measures quality of and stores DNA samples from each infant and both parents consented for the Pre-Vent Multicenter Study.

Clinical Research Centers (CRC). The Pre-Vent study consists of 5 Clinical Research Centers. Each CRC will conduct a site-specific

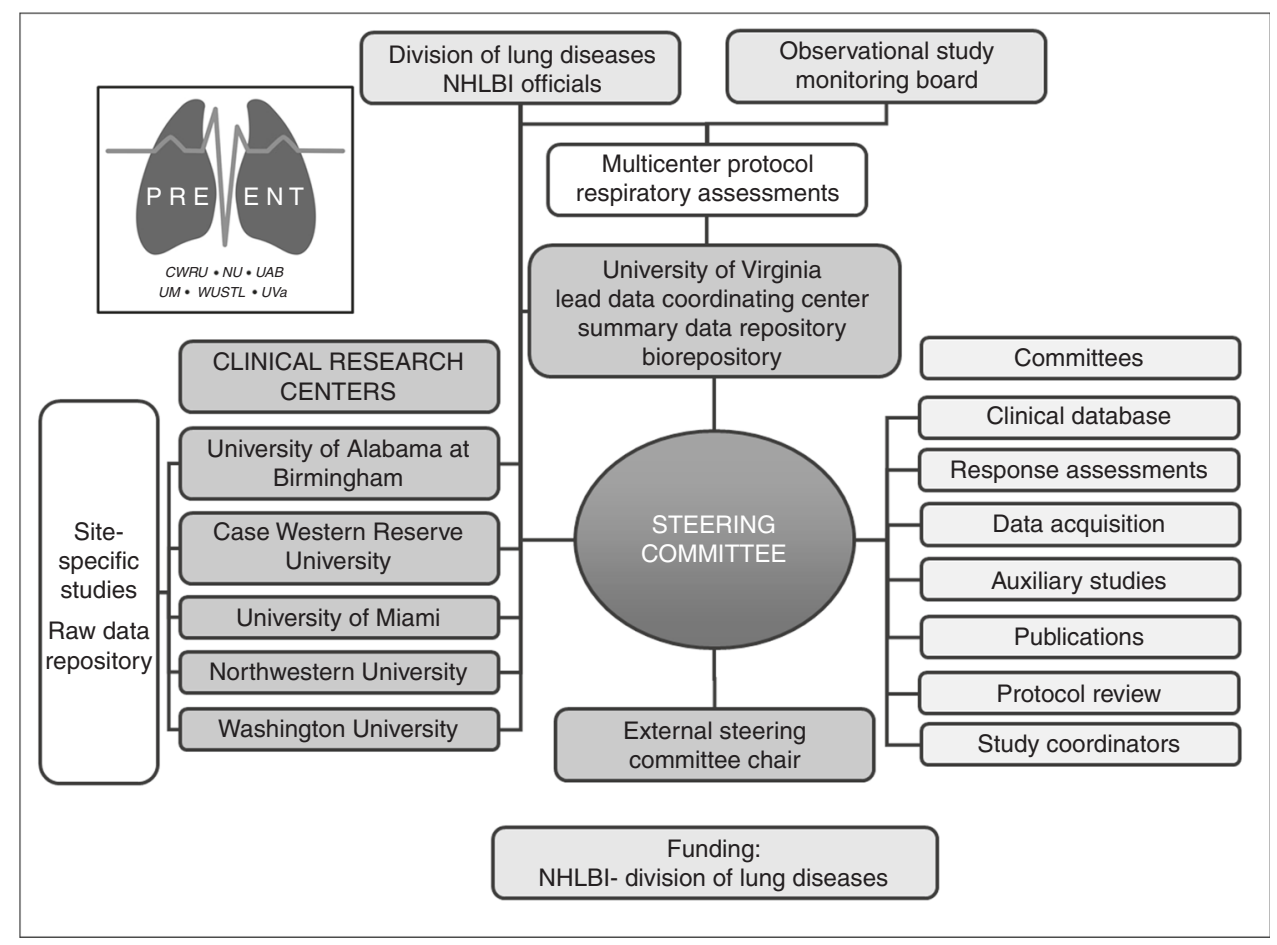

Fig. 1 Organizational chart for Pre-Vent 
Table 1. Inclusion and exclusion criteria for Pre-Vent enrollment

Inclusion criteria

$\bullet<29$ weeks gestational age

$\bullet<1$ week chronological age

Exclusion criteria

- Unlikely to survive or decision not to pursue full care

- Major congenital or chromosomal anomaly

study and contribute cardiorespiratory monitoring data for the collaborative multicenter protocol.

Committees. The LDCC organizes monthly Steering Committee teleconference meetings and twice yearly in-person meetings at the $\mathrm{NIH}$. The Clinical Database Committee developed the REDCap clinical database and addresses questions about data collection. The Data Acquisition Committee provided initial plans for storing the cardiorespiratory monitoring (CRM) data locally and distributing analytics, and continually monitors these processes to ensure valid data collection. The Auxiliary Studies Committee established a policy for reviewing and approving proposed auxiliary studies. The Publications Committee developed guidelines for authorship and a process for review and approval of manuscripts and abstracts prior to submission for publication or presentation. The Physiologic Response Assessments Committee holds in-person and teleconference meetings of the centers performing physiologic response testing in order to harmonize and standardize all tests and data collection performed at more than one center. The Protocol Review Committee reviews all proposed modifications in the universal and site-specific protocols and makes recommendations to the Steering Committee. Lastly, the Study Coordinators Committee holds conference calls discussing issues relating to the Research Coordinators from each center.

Multicenter study

The objective of the Pre-Vent Multicenter Study is to develop a predictive model of short-term respiratory outcomes in extremely preterm infants, based on quantitative analysis of NICU cardiorespiratory monitoring data.

Protocol. The multicenter prospective observational study includes $>500$ preterm infants $<30$ wks with at least $90 \%<$ 29 weeks. Inclusion criteria include $<1 \mathrm{wk}$ chronological age at the time of enrollment. Exclusion criteria include infants with congenital or chromosomal anomalies and those who are unlikely to survive (Table 1). The protocol uses only standard of care clinical measurements (i.e., bedside electrocardiogram, heart rate, respiratory rate, pulse oximetry), questionnaires and buccal DNA collection to provide minimally intrusive, highly detailed data collection on a very large cohort. Figure 2 is a timeline of the protocol. Waiver of consent was approved by the UVA IRB and three of the five CRC IRBs for standard of care clinical measurements while two CRCs preferred to use consent forms. All CRCs acquired consent for questionnaire and buccal DNA collection.

\section{Data collection, management, and analysis}

Clinical data: Using data collection forms designed by the Clinical Database Committee, the UVA Clinical Trials Office created a centralized REDCap database system, project-redcap.org. Each CRC contributes local data daily using a web-based interface. Maternal and infant medical records are reviewed for demographics, daily respiratory support and medications, nutritional support, neurologic imaging, retinopathy of prematurity outcomes, and other data related to common preterm morbidities.
An inpatient questionnaire is used to collect family history of asthma, allergies, eczema, sudden infant death, cyanosis, and snoring. A 52 weeks post-menstrual age (PMA) questionnaire is performed including clinical, medication, nutrition, environmental and respiratory information.

Individual centers retain constant access to their own data. In addition, all centers maintain an independent, customized, local REDCap database that is specific to their single CRC data acquisition including a mirror of the central REDCap database. The centralized database allows for real-time monitoring of Multicenter data entry by the UVA Clinical Trials Office in conjunction with the LDCC, while the CRCs' mirror databases allow easy integration of Multicenter data with CRC-specific data at the local level, without requiring double data entry.

Cardiorespiratory monitoring data: The LDCC coordinates data collection at each CRC which involves customized management and analysis. A summary highlighting differences among CRCs is shown in Table 2. Conversion to a common data format is the first and nontrivial step in distributing analytic tools. Algorithms developed for UVA and other specific systems may need to be modified to adjust for variations in sample frequency, data resolution, and amount of noise in the measurements.

This is a Big Data project-each infant produces up to $200 \mathrm{MB}$ per day of data from the bedside monitor, and we estimate the entire data set to be tens of TB. The Pre-Vent group is taking a new approach to multicenter Big Data collaborative studies. Rather than sending all the data to a central site for analysis, the LDCC will provide analytics software to the CRCs. The advantages are that data remain securely local while the analyses are performed in identical fashion at each CRC.

In addition to the sheer size of the signal data, the variety of monitors, data collection systems, and IT infrastructure across the five CRCs presents many challenges. To address this issue, the LDCC has developed a common file format to store the waveform and vital sign data as well as a GUI to allow remote CRCs to visualize these data in a common way.

Quantitative breathing record. LDCC investigators have developed numerical tools for quantitative detection of immature breathing (Fig. 3). This allows us to identify, count, and characterize episodes of central apnea and periodic breathing. Development plans included validation of $>100$ events by multiple clinicians with $>90 \%$ diagnostic accuracy. Prior to Pre-Vent, these tools were used to investigate central apnea, periodic breathing, cardiorespiratory interaction, and stochastic models in a large group of premature infants. ${ }^{11-15}$ The major findings were that apnea and periodic breathing have distinct time courses, increased apnea was associated with acute but not chronic illness, periodic breathing, if very prolonged, was sometimes associated with morbidity, and that the heart and lungs were progressively more coupled with post-natal development. In addition to metrics calculated directly from the waveforms, LDCC algorithms will calculate features from the discrete vital signs data including, for example, counts of events of intermittent hypoxemia and crosscorrelation between heart rate and $\mathrm{SpO}_{2} .{ }^{11}$ These tools will be used to analyze cardiorespiratory monitoring data from the $5 \mathrm{CRCs}$ and new algorithms developed at the LDCC or CRCs can also be tested.

Analytical approaches. Analyses will be conducted using observational research designs. The primary statistical model formulation will incorporate predictor variables from cardiorespiratory monitor data to detect and characterize immature breathing patterns. The products are statistical models to estimate the state of maturation and to predict future burdens of immature breathing. Precision of estimates from the statistical model of burden of apnea (and other states of immature breathing) will be 
a

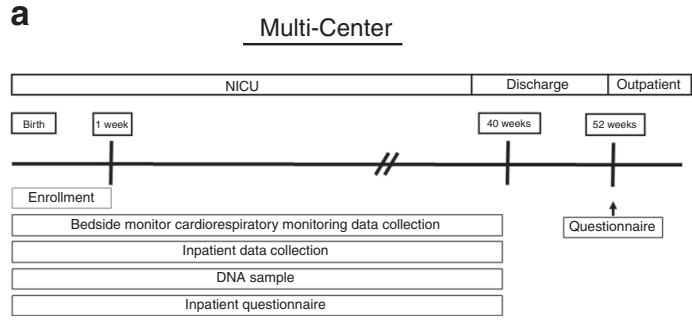

b

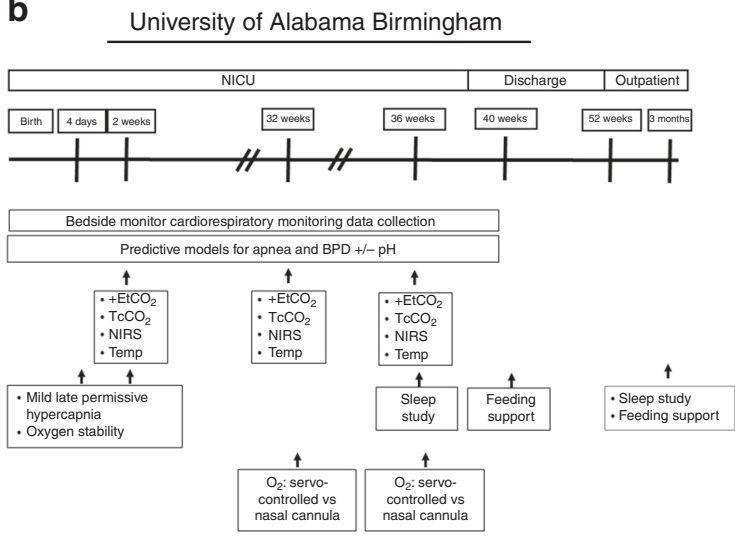

c

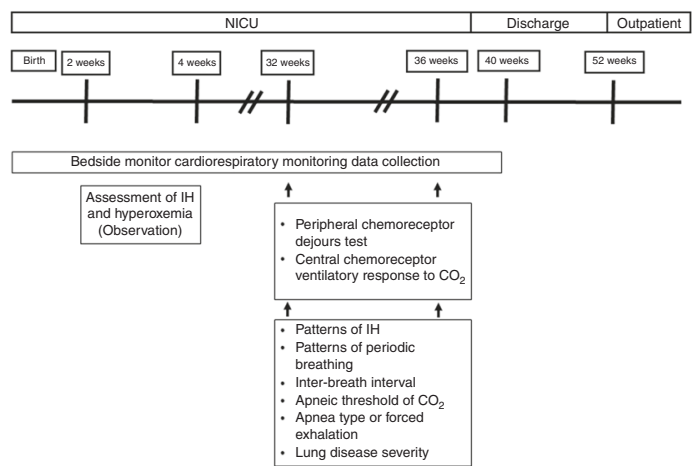

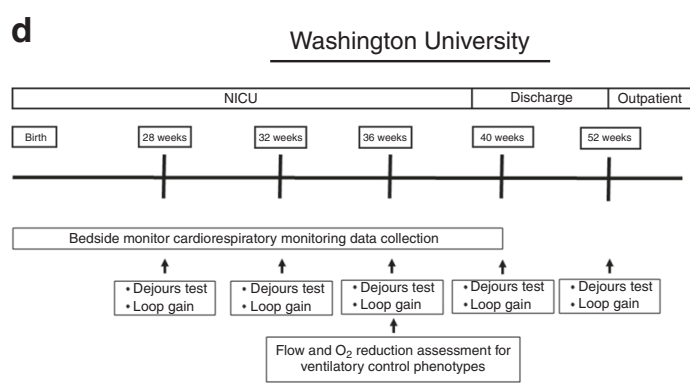

e

e Case Western Reserve University

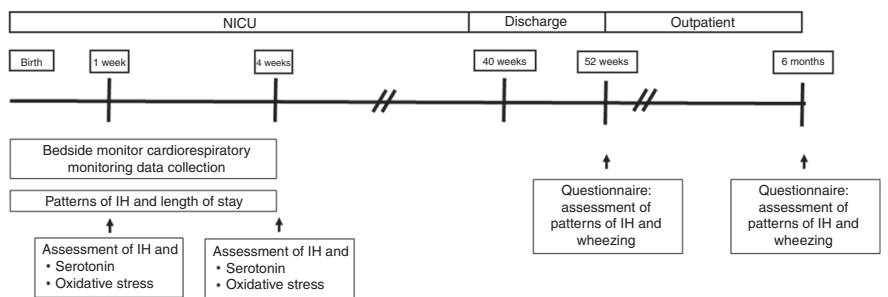

f

Northwestern University

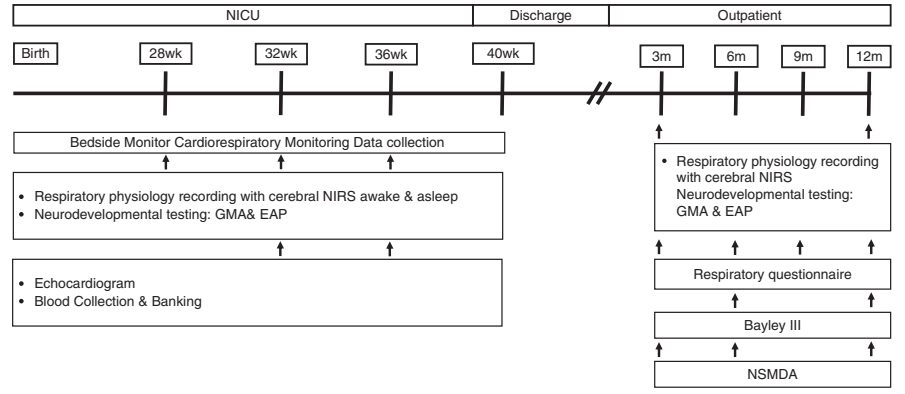

GMA: General Movements Assessment
EAP: Evoked Auditory Potential Trials

Bayley III: Bayley Scale of Infant \& Toddler Development III
NSMDA: Neurological, Sensory, \& Motor Development Assessment

Fig. 2 Timeline for data collection in the Pre-Vent multicenter (a) and single center protocols (b-f)

measured by the bias, standard error, mean-squared error, and the coefficient of determination ( $r$-squared). The statistical performance of the developed models will be assessed from multiple perspectives, by applying the developed models in independent validation populations within and between CRCs. The calibration of the model predictions will be assessed across increments of the full range of predicted values by comparing observed to expected cases, for the purpose of describing ranges of prediction where the model predictions are off-calibrated. Discrimination will be assessed using receiver operating characteristic curves. The proportion of the variability explained will be assessed by comparing the global model log-likelihood obtained for models excluding all covariate effects to that obtained with all included covariates.

Outcomes. There are two primary outcomes for the Pre-Vent multicenter protocol. The primary clinical outcome is respiratory status at 40 weeks PMA classified as unfavorable (receiving supplemental oxygen or flow/pressure respiratory support or respiratory medications), or favorable (none of the unfavorable conditions).
The primary physiological outcome is a quantitative measure of immature breathing patterns (central apnea and periodic breathing) and cardiorespiratory events (hypoxemia, bradycardia), as plotted on a standard curve of scores for gestational age-matched infants at 36-37 weeks PMA. This fulfills a central goal, to define what is normal in terms of frequency and severity of apnea and cardiorespiratory instability events (bradycardia, hypoxemia) across a spectrum of gestational and post-menstrual ages, modes of ventilatory support, and supplemental oxygen. Knowing the ranges of normal will facilitate studies relating exaggerated immature breathing patterns or cardiorespiratory instability events with chronic respiratory dysfunction and other adverse outcomes in extremely preterm infants. From legacy data the LDCC has fashioned a clinical tool based on the familiar infant growth chart (Fig. 4). The new infant breathing chart plots percentiles as a function of age for the number of apnea $\geq 10 \mathrm{sec}$ accompanied by bradycardia and intermittent hypoxemia (top), and the proportion of time spent in periodic breathing in 355 infants $<29$ wks GA (bottom). The anticipated use is that of the growth curve-events are measured from analysis of the monitor data, and the results are compared to the chart. A larger than 
Table 2. Summary highlighting differences among CRCs

\begin{tabular}{lllllll}
\hline CRC & Monitor & Data collection system & ECG (Hz) & Respiratory (Hz) & Block size (seconds) & SpO ${ }_{2} \%$ Res \\
\hline Case Western Reserve University & SpaceLab & LabView & 200 & 200 & 1 & 0.1 \\
University of Miami & Philips & MediCollector/Data Warehouse & 250 & 62.5 & 1.024 & 0.1 \\
Northwestern University & Philips & MediCollector/BedMaster & 250 & 62.5 & 1.024 & 1 \\
Washington University & Philips & BedMaster & 250 & 62.5 & 1.024 & 1 \\
University of Alabama Birmingham & Philips & BedMaster & 125 & 125 & 1 \\
\hline
\end{tabular}
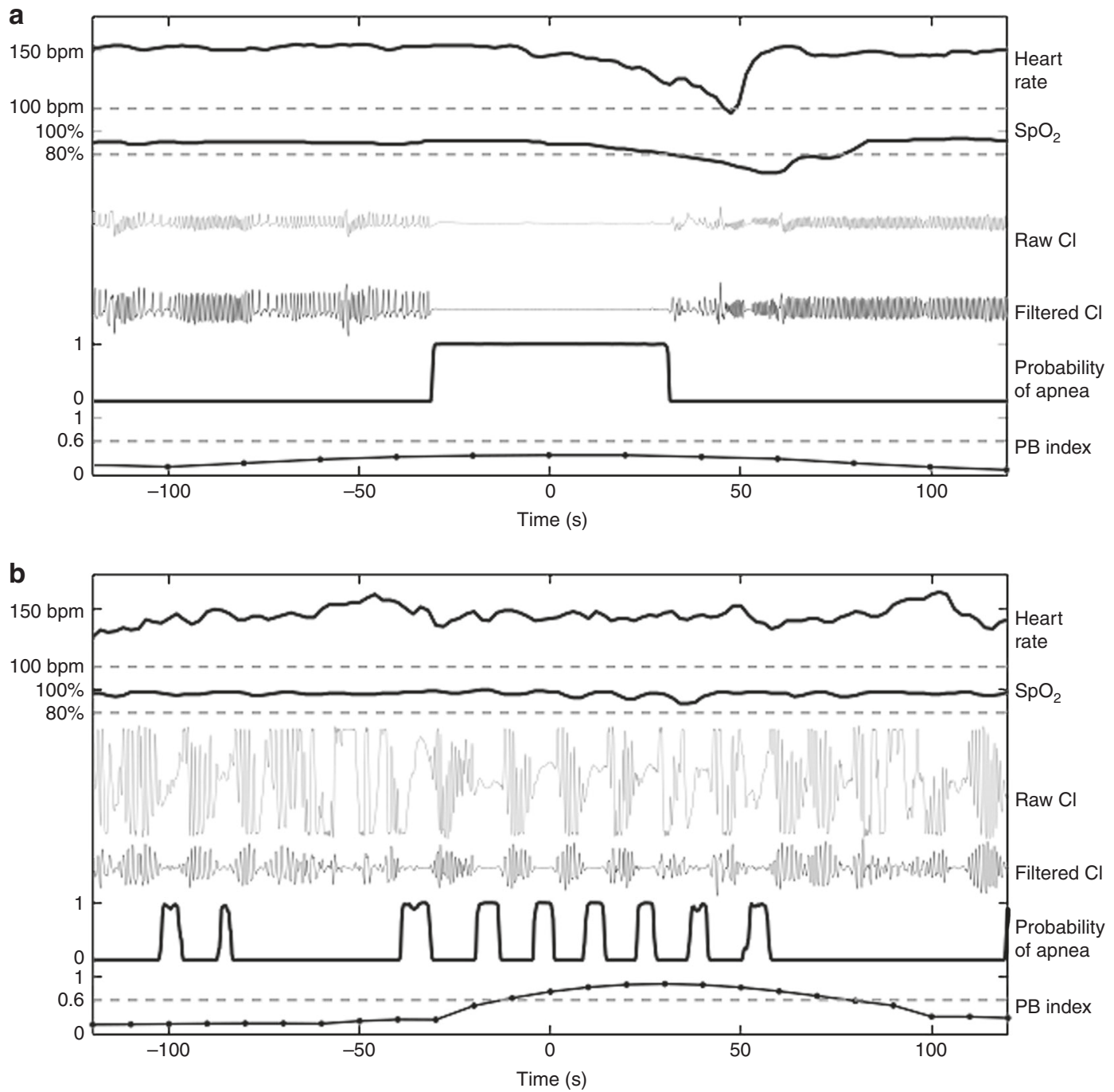

Fig. 3 A quantitative breathing record for an episode of central neonatal apnea (a) and of periodic breathing (b). In each, the top three tracings are as shown on the bedside monitor: heart rate, oxygen saturation $\left(\mathrm{SpO}_{2}\right)$ and chest impedance $(\mathrm{Cl})$. The bottom three time series are generated by numerical algorithms developed at the College of William and Mary whose results were validated clinically: filtered $\mathrm{Cl}$, probability of apnea and an index of periodic breathing (PB). In (a), the long apnea is accompanied by bradycardia (heart rate 100) and desaturation $\left(\mathrm{SpO}_{2}<80 \%\right)$, and the PB index remains below a threshold for detection. In (b), the seven regularly spaced apneas lead to neither bradycardia nor desaturation, but the PB index exceeds the threshold for detection

expected rate of events might lead to reconsideration of the respiratory support of the infant, and an acute increase might lead to consideration of subacute potentially catastrophic illnesses such as sepsis or necrotizing enterocolitis.

Secondary respiratory outcomes include prolonged immature control of breathing, time on respiratory support and medications, chronic lung disease, wheezing, and pulmonary hypertension. Infants will be followed until NICU discharge and 52 weeks PMA.
Non-respiratory secondary outcomes will also be assessed in relation to apnea and chronic lung disease, including sepsis, necrotizing enterocolitis, retinopathy of prematurity, and neurologic outcomes. Every record is inspected for the means of respiratory support, and clinical events of sepsis or necrotizing enterocolitis, rather than relying on database queries that are often incomplete or inaccurate. We anticipate that this approach will result in more accurate event identification, making predictive 

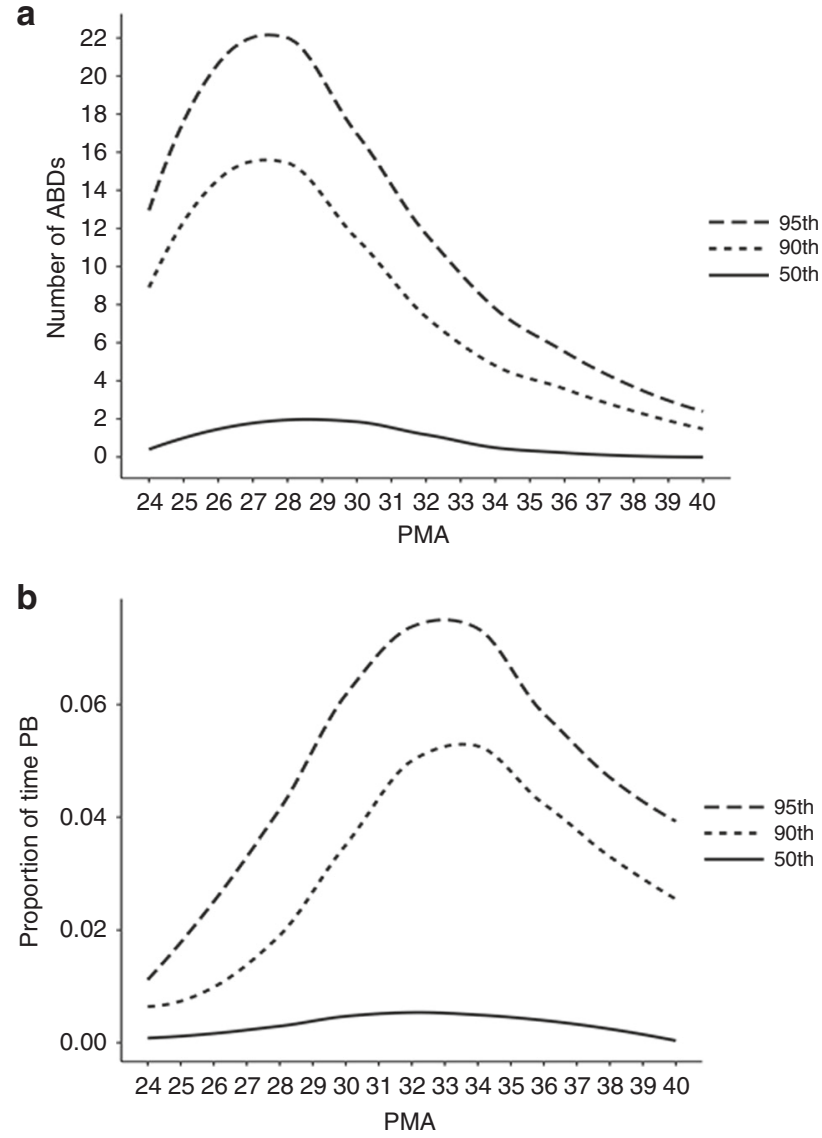

Fig. 4 Percentile chart for control of breathing. Data from 355 infants of EGA $<29$ weeks are represented. Each line marks the designated percentile $\left(95^{\text {th }}, 90^{\text {th }}, 75^{\text {th }}\right.$, and $\left.50^{\text {th }}\right)$ of number of apnea $>10 \mathrm{sec}$ with accompanied bradycardia and hypoxemia, measured as $\mathrm{O}_{2}$ desaturation (ABD, top panel, a) or epochs of periodic breathing (bottom panel, b). Thus, infants in this EGA category have a median of fewer than 2 ABD events, peaking around week 28 postmenstrual age, and more than 14 events would be very abnormal. Likewise, they have a median of less than $1 \%$ total time in periodic breathing, peaking around week 34 , and more than $7 \%$ would be very abnormal

algorithms more robust in real-world use. Thus, an important product of Pre-Vent is an annotated database of clinical outcomes matched to cardiorespiratory monitoring data.

Clinical research center studies

Each CRC will contribute cardiorespiratory monitoring data for the collaborative multicenter study as previously discussed. In addition, each CRC will perform center-specific protocols with the corresponding timelines shown in Fig. 2.

University of Alabama Birmingham. The overall goal of the research project is to determine in individual extremely preterm infants the risk for hypoxemic and/or bradycardic episodes and longer-term outcomes such as BPD, BPD with pulmonary hypertension, and feeding support at three months corrected age, and identify interventions that may prevent or reduce such outcomes. This protocol seeks to develop and validate mathematical models of ventilatory patterns based on multiparametric vital signs ( $\left.\mathrm{HR}, \mathrm{RR}, \mathrm{SpO}_{2}\right)$ monitoring and signal analysis methods to predict hypoxemic and/or bradycardic episodes in 100 individual infants before they occur (on multiple time scales ranging from seconds to hours) and identify patterns of ventilatory abnormalities associated with BPD, BPD associated with pulmonary hypertension, as well as feeding support at three months corrected age. An additional goal is to determine if late mild permissive hypercapnia (increasing $\mathrm{TcCO}_{2}$ by $5 \mathrm{~mm} \mathrm{Hg}$, while maintaining $\mathrm{pH}>7.2$ ) is associated with reduction in apnea, bradycardia, and hypoxemic episodes and improved stability of oxygenation. Finally, this proposal seeks to determine if randomization to a servo-controlled oxygen environment versus oxygen administered by nasal cannula is associated with reduction in $\mathrm{IH}$ and improved oxygen stability. Algorithms ${ }^{16,17}$ that are being developed will be employed to predict and assess the risk in enrolled preterm infants.

Case Western Reserve University. The primary objective of this protocol is to assess the association between patterns of $\mathrm{IH}$ (i.e., frequency, duration, severity, and timing) and clinical outcomes ${ }^{18}$ in 150 infants $\leq 306 / 7$ wks GA focusing on NICU length of stay and wheezing up to 6 months corrected age. The secondary objective is to explore candidate biochemical markers associated with patterns of $\mathrm{IH}$, specifically serotonin metabolites, and markers of oxidative stress ${ }^{19}$ derived from serum and urine samples hypothesizing that $\mathrm{IH}$ events at one week and one month of age will be associated with decreased serotonin levels and increased markers of oxidative stress. The findings of this proposal may provide insight into specific patterns of $\mathrm{IH}$ that are associated with prolonged hospitalization and respiratory morbidity and identify possible $\mathrm{IH}$ induced mechanistic pathways that initiate a pathological cascade.

University of Miami. Extremely premature infants have persistent respiratory instability, which is likely due to an altered maturation of the control of breathing $(\mathrm{CoB})$ system. Experimental animal data indicates that $\mathrm{IH}$ and hyperoxemia during the neonatal period can alter the development of the CoB. This study will evaluate the extent to which exposure to $\mathrm{IH}$ and hyperoxemia, or both, during the neonatal period are associated with altered maturational trajectory and/or impaired function of the $C o B$ system in extremely premature infants. Ventilatory response to high inspired oxygen (Dejours test) and inspired carbon dioxide will be evaluated to assess peripheral and central chemoreceptor function at 32 and 36 wks PMA in 105 premature infants of 23-28 wks gestational age. ${ }^{20}$ These data will be used to delineate the maturational trajectory of their $\mathrm{COB}$ system and will be correlated to the degree of exposure to $\mathrm{IH}$ and hyperoxemia during the $2^{\text {nd }}$ through $4^{\text {th }}$ post-natal weeks. This study will also determine the extent to which alterations in CoB function are associated with respiratory and oxygenation instability at near term corrected age. Lastly, the study will determine the prevalence of different mechanisms of $\mathrm{IH}$ and examine their contribution to respiratory instability in this population. This study will provide new insight into the etiology of the persistent respiratory instability in this population and will improve our understanding of the consequences of abnormal oxygen levels during the neonatal period. The findings of this investigation may also open new avenues to develop novel strategies to improve respiratory stability in this vulnerable population.

Northwestern University. This protocol will use comprehensive, state-of-the-art, high-fidelity monitoring to investigate physiological biomarkers of autonomic neurorespiratory maturation with integrated analysis of autonomic nervous system (ANS) responses in preterm infants, and evaluate their role in ventilatory instability, BPD, and co-morbidities in the first year of life. High-resolution, integrated physiologic monitoring will be performed at: 28, 32, and 36 wks PMA, and at 3 and 12 months corrected age. These recordings will last up to $24 \mathrm{~h}$ at the NICU recording time points $(28,32$, and 36 wks PMA) and $4 \mathrm{~h}$ for the outpatient testing time points. High-resolution physiologic recordings are used to establish the spectrum and developmental trajectory of ANS 
maturation and function throughout the first year. Recorded signals will include: electrocardiogram (ECG), respiratory inductance plethysmography (RIP) of chest and abdomen, individually and summed, $\mathrm{SpO}_{2}$ and pulse waveform, end tidal and transcutaneous carbon dioxide $\left(\mathrm{EtCO}_{2}\right.$ and $\left.\mathrm{TcPCO}_{2}\right)$ and waveform, blood pressure (BP), cerebral near-infrared spectroscopy (cNIRS), as well as sleep state staging measures including electroencephalogram (EEG), electromyogram (EMG), and electro-oculogram (EOG). Establishing the role of autonomic neurorespiratory maturation in stability of oxygenation and ventilation throughout the first year of life will provide insight into pathogenesis of chronic respiratory disease, allow prospective identification of at-risk infants, and permit development of mechanism-specific interventions.

Washington University. This protocol seeks to leverage the continuously recorded oxygen saturation and analyze the impact of hypoxia and hyperoxia, whether intermittent or continuous, on the maturation of the components of the ventilatory control apparatus, as manifest by loop gain and carotid body activity function in 100 to 110 preterm infants. At 36 weeks physiologic tests will be performed to assign one of 4 ventilatory phenotypes to each infant tested. The multi-dimensional phenotype of bronchopulmonary dysplasia/chronic lung disease will be defined by passing or failing a physiologic test in which infants on supplemental $\mathrm{O}_{2}$ breathe room air and infants already on room air breathe a mildly hypoxic gas. Earlier studies ${ }^{9}$ suggest that these physiologic tests can determine the relative contributions of immature ventilatory control mechanisms and parenchymal lung disease to the infant's ability to maintain respiratory pattern, rate and oxygen saturation. A sample size of $\sim 100$ will allow for more rigorous exploration and to establish the association, if any, between loop gain and the potential for respiratory pattern instability. In addition, the transient hyperoxia test ${ }^{21}$ will be used to determine the contribution of the peripheral chemoreceptors to ventilatory drive. Potential correlations between spontaneously occurring intermittent hypoxemia events during development and peripheral chemoreceptor function, loop gain or respiratory pattern instability will also be explored. These analyses will allow for identification of infants requiring supplemental $\mathrm{O}_{2}$ or additional support because of immature respiratory control. Prolonged polysomnographic recordings will not be needed. Rather, brief studies during quiet sleep, with sigh analysis will allow for identification of infants with strong propensities for respiratory pattern instability.

Interactions of Clinical Research Center and Multicenter protocols. The Multicenter study and CRC studies share a common goal of investigating mechanisms of ventilatory control (e.g., chemoreceptor, mechanoreceptor, developmental, etc.) that contribute to instability of oxygenation and risk of morbidity and mortality in premature infants. Multicenter data in the central REDCap database and the central storage of CRM result files are readily available by request to each CRC for use in their 5 approved CRC studies. Data and specimens relevant only to a CRC's studies will be entered and maintained locally. CRCs can recruit additional infants for their approved CRC studies, including some born between 29-30 weeks per the FOA, and can obtain additional history, physiologic response tests, biospecimens, or other data not collected by the Multicenter consortium. CRCs are encouraged to share this local data with other CRCs. Multicenter data may be available to Pre-Vent investigators and other investigators for studies outside the scope of the $5 \mathrm{NIH}$-approved CRC studies by applying through the Pre-Vent Auxiliary Studies Committee.

Biomedical sample repository. The goal of the DNA biorepository is to provide a source of DNA from subjects and their parents for future investigation of genetic and epigenetic contributions to adverse outcomes or resilience following birth at extremely low gestational ages. The Pre-Vent DNA collection will add to the availability specimens that are linked with highly phenotyped, extremely low birthweight infants in order to investigate the genetic basis of prematurity and its associated consequences. The LDCC in conjunction with the biorepository (UVa Biorepository and Tissue Research Facility) established standardized procedures for cross-sectional DNA collection (buccal swabs), processing, and central storage. The Pre-Vent Auxiliary Studies Committee formalized a protocol to allow Pre-Vent related researchers to request DNA samples while stored at the Biorepository and Tissue Research Facility based on justification and feasibility of the proposed assays, and independent funding to analyze the samples and the resulting data. ${ }^{22}$ At the end of the study, DNA will be transferred to NHLBI's BioLINCC, ${ }^{23}$ and access will be allowed under BioLINCC's review process.

\section{DISCUSSION}

The aim of the Pre-Vent Multicenter Study is to develop a predictive model of short-term respiratory outcomes in extremely preterm infants, based on quantitative analysis of NICU cardiorespiratory monitoring data. The study is a large-scale epidemiologic analysis that will serve to develop a personalized quantitative breathing record and also provide normative data for this cohort of vulnerable infants at risk of respiratory complications. It will also be an outstanding platform for sharing analytics as this will be the first study where the data gathered will be available at each CRC rather than at a centralized data repository.

The overall charge of Pre-Vent is to elucidate the interactions between immature control of breathing and chronic respiratory morbidities in preterm infants. Utilizing cardiorespiratory monitoring waveform data collected at each center, the five collaborating centers will work together to characterize how multiple, seemingly unconnected components of immature respiratory control contribute to later pulmonary and neurodevelopmental morbidity. Three of the centers will further investigate the resilience and function of the central and peripheral components of the respiratory control system in these infants, and others will evaluate biomarkers to understand indices of autonomic dysfunction.

This use of Big Data techniques offers the opportunity to gain insights into the pathogenesis of respiratory disorders of prematurity and discovery of new targets for prevention and treatment as well as serve as a predictor of respiratory disease. Mathematical algorithms developed in Pre-Vent may lead to standardized interpretation of data and identification of optimal interventions based on specific identified cardiorespiratory patterns. For example, clinicians can plot the numbers of apnea, bradycardia and intermittent hypoxemia events and the burden of periodic breathing on the gestational quantitative breathing curves for individual infants, and gain insight into the rate of maturation of respiratory control, the mechanism of respiratory dependency (i.e., ventilatory control vs. lung or airway disease) and the need for a specific modality of respiratory support (i.e., respiratory stimulant, diaphragmatic triggering, pressure support, oxygen, or bronchodilator) in the near future. Identification of specific cardiovascular, respiratory, and ventilatory phenotypes from the analysis of large amounts of continuously recorded data would help interpret the significance of apneas, bradycardias, and desaturations in premature infants and could ultimately lead to personalized therapies.

\section{ACKNOWLEDGEMENTS}

We thank D.E. McKaig and J.B. Delos, College of William and Mary, for Fig. 4. The National Institutes of Health (NIH) and the National Heart, Lung, and Blood Institute (NHLBI) provided grant support. NHLBI staff had input into the study design, conduct, 
analysis, and manuscript drafting. The content is solely the responsibility of the authors and does not necessarily represent the official views of the $\mathrm{NIH}$. Participating sites collected and stored the data. The site Pls had full access to individual site data and take responsibility for the integrity of the waveforms. The lead data coordinating center analyzed the data. Drs. Moorman and Lake take responsibility for the integrity of the data and accuracy of the data analysis. We are indebted to our medical and nursing colleagues and infants and parents who agreed to take part in this study. The following investigators participated in this study: Providence, RI: P.A. Dennery, Brown University, Rhode Island Hospital, Hasbro Children's Hospital Bethesda, MD: A.D. Laposky, A. Natarajan, B.Schmetter., J. Troendle; National Institutes of Health, National Heart, Lung and Blood Institute. Charlottesville, VA: J.R. Moorman, D. Lake, K. Nash Krahn, A.M. Zimmet, A.K. Camblos, S.A. Fowler, K.D. Fairchild, A.A. Flower, P. Pramoonjago, C.A. Rumpel; University of Virginia. Cleveland, OH: A.M. Hibbs, R.J. Martin, J.M. Di Fiore, T. Raffay, P.M. MacFarlane; Case Western Reserve University, University Hospitals Cleveland Medical Center, Rainbow Babies and Children's Hospital A. Zadell, University Hospitals Cleveland Medical Center, Rainbow Babies \& Children's Hospital C. Tatsuoka, Case Western Reserve University. Valencia, Spain: M. Vento, University and Polytechnic Hospital La Fe, Health Research Institute La Fe. Chicago, IL: A. Hamvas, D. Weese-Mayer, R.A. DeRegnier; Northwestern University, Ann \& Robert H. Lurie Children's Hospital of Chicago and Stanley Manne Children's Research Institute A. Bradley, M. Carroll, E. Dunne, S. Fair, B. Hopkins, C. Rand, M. Schau; Ann \& Robert H. Lurie Children's Hospital of Chicago and Stanley Manne Children's Research Institute C.R. Estabrook, Northwestern University. Birmingham, AL: N. Ambalavanan, A. Nakhmani, W.A. Carlo, D. Laney, B. Troxler, C.P. Travers; University of Alabama. P. Indic, University of Texas Tyler, Tyler, TX; University of Alabama. Austin, TX: D. Paydarfar, University of Texas Austin. Worcester, MA: E. Salisbury, University of Massachusetts. Miami, FL: E. Bancalari, N. Claure, A.C. Aguilar, C. D'Ugard, D. Jain, D. Ludwig, A. Schott, S. Vanbuskirk; University of Miami, Holtz Children's Hospital - Jackson Memorial Medical Center. St. Louis, MO: J. Kemp, R. Colvin, B. Bellm, M. McLeland, J. Hoffmann, K. Schechtman, J. Shimony, C. Smyser, L. Linneman, J. Hoover, B. Warner; Washington University. J. Egan, H. Pyles; St. Louis Children's Hospital. Little Rock, AR: J.L. Carroll, University of Arkansas. Melbourne, Australia: B. Edwards, Monash University. Supported by NIH grants U01 HL133708, U01 HL133643, U01 HL133704, U01 HL133536, U01 HL133689, U01 HL133700.

\section{AUTHOR CONTRIBUTIONS}

P.A.D., J.M.D.F., N.A., E.B., J.L.C., N.C., A.H., A.M.H., P.I., J.K., K.N.K., D.L., A.L., R.J.M., A.N., C.R., M.S., D.E.W.-M., A.M.Z., and J.R.M. provided substantial contributions to conception and design, and proposed acquisition of data, or analysis and interpretation of data; P.A.D., J.M.D.F., N.A., E.B., J.L.C., N.C., A.H., A.M.H., P.I., J.K., K.N. K., D.L., A.L., R.J.M., A.N., D.E.W.-M., A.M.Z., and J.R.M. participated in drafting the article or revising it critically for important intellectual content; and P.A.D. provided final approval of the version to be published

\section{ADDITIONAL INFORMATION}

Competing interests: DL has a small equity share $(<1 \%)$ in Medical Predictive Science Corporation, which markets the HeRO heart rate monitoring system. JRM is Chief Medical Officer and shareholder, Advanced Medical Predictive Devices, Diagnostics and Displays, and shareholder, Medical Predictive Science Corporation, both in Charlottesville, VA. All other authors declare no competing interests.

Publisher's note: Springer Nature remains neutral with regard to jurisdictional claims in published maps and institutional affiliations.

\section{REFERENCES}

1. Stoll, B. J. et al. Trends in care practices, morbidity, and mortality of extremely preterm neonates, 1993-2012. JAMA 314, 1039-1051 (2015).

2. Bancalari, E. \& Jain, D. Bronchopulmonary dysplasia: can we agree on a definition? Am. J. Perinatol. 35, 537-540 (2018).

3. Ren, C.L. et al. Tidal breathing measurements at discharge and clinical outcomes in extremely low gestational age neonates. Ann. Am. Thora. Soc. 15, 1311-1319 (2018).

4. Bancalari, E. \& Claure, N. Respiratory instability and hypoxemia episodes in preterm infants. Am. J. Perinatol. 35, 534-536 (2018).

5. Darnall, R. A. The role of $\mathrm{CO}(2)$ and central chemoreception in the control of breathing in the fetus and the neonate. Respir. Physiol. Neurobiol. 173, 201-212 (2010).

6. Eichenwald, E. C. Apnea of Prematurity. Pediatrics 137, e1-e7 (2016).

7. Schmidt, B. et al. Long-term effects of caffeine therapy for apnea of prematurity. New Engl. J. Med. 357, 1893-1902 (2007).

8. Rhein, L. M. et al. Effects of caffeine on intermittent hypoxia in infants born prematurely: a randomized clinical trial. JAMA Pediatr. 168, 250-257 (2014).

9. Coste, F. et al. Ventilatory control and supplemental oxygen in premature infants with apparent chronic lung disease. Archives of disease in childhood. Fetal Neonatal Ed. 100, F233-F237 (2015).

10. Poets, C. F. et al. Association between intermittent hypoxemia or bradycardia and late death or disability in extremely preterm infants. JAMA 314, 595-603 (2015).

11. Fairchild, K. D. \& Lake, D. E. Cross-correlation of heart rate and oxygen saturation in very low birthweight infants: association with apnea and adverse events. Am. J. Perinatol. 35, 463-469 (2018).

12. Clark, M. T. et al. Stochastic modeling of central apnea events in preterm infants. Physiol. Meas. 37, 463-484 (2016).

13. Fairchild, K. et al. Clinical associations of immature breathing in preterm infants: part 1-central apnea. Pediatr. Res. 80, 21-27 (2016).

14. Mohr, M. A. et al. Quantification of periodic breathing in premature infants. Physiol. Meas. 36, 1415-1427 (2015).

15. Patel, M. et al. Clinical associations with immature breathing in preterm infants: part 2-periodic breathing. Pediatr. Res. 80, 28-34 (2016).

16. Gee, A. H., Barbieri, R., Paydarfar, D. \& Indic, P. Predicting bradycardia in preterm infants using point process analysis of heart rate. IEEE Trans. bio-Med. Eng. 64, 2300-2308 (2017)

17. Amperayani VNSA, P. I., Travers, C. P., Barbieri, R., Paydarfar, D. \& Ambalavanan, N. An algorithm for risk stratification of preterm infants. Comput. Cardiol. 44, 1-4 (2017).

18. Di Fiore, J. M. et al. The relationship between patterns of intermittent hypoxia and retinopathy of prematurity in preterm infants. Pediatr. Res. 72, 606-612 (2012).

19. Nanduri, J. \& Prabhakar, N. R. Developmental programming of $\mathrm{O}(2)$ sensing by neonatal intermittent hypoxia via epigenetic mechanisms. Respir. Physiol. Neurobiol. 185, 105-109 (2013).

20. Gerhardt, T. \& Bancalari, E. Apnea of prematurity: I. Lung function and regulation of breathing. Pediatrics 74, 58-62 (1984).

21. Sovik, S., Eriksen, M., Lossius, K., Grogaard, J. \& Walloe, L. A method of assessing ventilatory responses to chemoreceptor stimulation in infants. Acta paediatrica (Oslo, Norway: 1992) 88, (563-570 (1999).

22. Pryhuber, G. S. et al. Prematurity and respiratory outcomes program (PROP): study protocol of a prospective multicenter study of respiratory outcomes of preterm infants in the United States. BMC Pediatr. 15, 37 (2015).

23. Giffen, C. A. et al. Providing contemporary access to historical biospecimen collections: development of the NHLBI Biologic Specimen and Data Repository Information Coordinating Center (BioLINCC). Biopreservation biobanking 13, 271-279 (2015). 